\title{
Oriented ultra-high molecular weight polyethylene/gold nanocomposites: Electrical conductivity and chain entanglement dynamics
}

\author{
S. X. Drakopoulos ${ }^{1 *}$, G. C. Psarras ${ }^{2}$, S. Ronca ${ }^{1}$ \\ ${ }^{1}$ Department of Materials, Loughborough University, LE11 3TU Leicestershire, United Kingdom \\ ${ }^{2}$ Smart Materials \& Nanodielectrics Laboratory, Department of Materials Science, University of Patras, 26504 Patras, \\ Hellas (Greece)
}

Received 25 August 2020; accepted in revised form 15 November 2020

\begin{abstract}
Entanglement dynamics of uniaxially-oriented, disentangled ultra-high molecular weight polyethylene nanocomposites modified with gold nanoparticles are investigated, using dielectric spectroscopy, during the transition to melt state. The dc conduction is approximately calculated via the logarithmic derivative of dielectric permittivity and is observed to decrease with the formation of entanglements. As the draw ratio increases, the progressive formation of entanglements resulted in a stronger dc conductivity decrease due to the loss of orientation in the pre-melt crystalline and partially oriented amorphous chain segments. Additionally, a sharp peak is obtained in the absence of dc conductivity, attributed to the dipolar contribution of the Maxwell-Wagner-Sillars (MWS) interfacial polarization between the gold nanoparticles and the polymer chains. The relaxation time of the MWS interfacial polarization increases with the progressive formation of entanglements, as observed in a previous study. The results presented shed light on the process of entanglement formation in oriented ultrahigh molecular weight polyethylene nanocomposites.
\end{abstract}

Keywords: nanocomposites, disentangled ultra-high molecular weight, uniaxial orientation, broadband dielectric spectroscopy, entanglement dynamics

\section{Introduction}

Scientists and engineers face obstacles to control the melt-processing of ultra-high molecular weight polyethylene (UHMWPE) with conventional methods due to chain entanglements, causing extremely high viscosity $[1,2]$. The introduction of synthetic methods based on single-site catalysts has allowed the production of low-entanglements-density polyethylene material (dis-UHMWPE), showing improved processing and manufacturing features [3, 4]. This concept has also been observed via Scanning Electron Microscopy (SEM), where the dis-UHMWPE sample exhibits a 'rose flower' morphology, with petals originating from crystalline lamellae [5]. The thickness of said lamellae is about $25 \mathrm{~nm}$ that, according to the Gibbs-Thomson equation for polyethylene $\left(T_{\mathrm{m}}=414.2-259.7 / 1\right)$, should correspond to a melting temperature of about $131{ }^{\circ} \mathrm{C}[6,7]$. However, nascent dis-UHMPE has a considerably higher melting temperature $\left(\sim 140^{\circ} \mathrm{C}\right)$, where the value drops to the expected one only after the sample has experienced a melting-recrystallization cycle. This observation has been attributed to the fact that nascent UHMWPE crystals, especially for dis-UHMWPE, are formed by few chains, with many consecutive re-entries of the same chain in one crystal. These tight folds increase the energy needed to melt the crystal. When the nascent crystals are molten and re-crystallized, 
the crystals formed from the entangled melt do not present consecutive re-entries, as they are instead formed by many chains' segments randomly entering the various crystals. Several papers have discussed this aspect through DSC experiments [8-10]. The disentangled character is, however, a metastable condition for the material. Once the melt state is reached above $140^{\circ} \mathrm{C}$, the mobility of the polymer chains increases, resulting in an increase in chain reptation. With the evolution of time, the metastable state transitions towards the thermodynamically favored, fully entangled configuration. We have recently reported that, in principle, entanglements are energetically favorable in the disentangled amorphous parts of the polymer at temperatures just above $55^{\circ} \mathrm{C}$, but the process is kinetically hampered by the presence of crystals [11]. Rheological and dielectric studies have shown that the values of the real part of modulus (mechanical and electric respectively) of dis-UHMWPE increase with time, approaching the equilibrium value in the fully entangled state, as expected from the progressively reduced mobility of the increasingly entangled chains $[11,12]$.

The molecular dipolar fluctuations, thermal transitions, charge transport, and interfacial phenomena as a function of frequency and temperature in polymeric materials can be monitored by means of broadband dielectric spectroscopy (BDS) [13]. Dielectric data can be analyzed employing different formalisms, such as electric modulus, dielectric permittivity, impedance, and ac conductivity [14]. Depending on the specific phenomena under study, a particular formalism could prove more effective in providing information for the physical mechanisms of interest $[15,16]$.

Polyolefins like polypropylene and polyethylene are being employed as dielectric materials for capacitive electrical energy storage applications due to their highly insulating character $[17,18]$. The use of polymers in such applications offers advantages in terms of mechanical, lightweight, and processing properties $[19,20]$. To enhance the dielectric properties of polymers, nanofillers of various shapes (nanoparticles, nanofibres, and nanoplates) and electrical characteristics (from insulating to conducting) have been employed [21]. Gold nanostructures, for example, are used as fillers to increase electrical conductivity and hence increase the charge flow and energy recovery speed in supercapacitor applications [22, 23].

In general, polymers build up heat due to their very low thermal conductivity; however, it has been shown that semicrystalline polymers, where high orientation is induced through processing, are able to dissipate more efficiently thermal energy through lattice movement [24, 25]. Recently [26-28], it has been reported that oriented dis-UHMWPE exhibits an increase in thermal conductivity of about 10 to 200 times, depending on the draw ratio: this could potentially open the possibility to dielectric materials with a better life performance.

In the present work, we have followed the entanglements formation, applying the electric modulus formalism and dc conduction to uniaxially oriented disUHMWPE/gold nanocomposites progressively transitioning to the melt state. The dc conduction values are calculated via the logarithmic derivative of dielectric permittivity as an approximation from the ac spectrum. A strong peak is obtained when the conductivity contribution is excluded due to the dipolar contribution of the Maxwell-Wagner-Sillars interfacial polarization between the gold nanoparticles and the polymer chains. The dc conduction is found to decrease significantly with the formation of entanglements, even more, when the higher draw ratio samples are considered, a phenomenon that can be attributed to the more severe transition from highly crystalline/highly ordered amorphous to fully disordered melt state.

\section{Experimental methods}

\subsection{Chemical synthesis of dis-UHMWPE}

The synthesis of dis-UHMWPE was done in-house following the procedure described elsewhere [29]. The polymerization reaction was quenched with acidified methanol (methanol $\left(\mathrm{CH}_{3} \mathrm{OH}\right) / 37 \% \mathrm{w} / \mathrm{w}$ hydrochloric acid $(\mathrm{HCl}) 95 / 5 \mathrm{v} / \mathrm{v}$ ) in order to avoid the formation of aluminum oxide $\left(\mathrm{Al}_{2} \mathrm{O}_{3}\right)$ catalytic ashes from the methyaluminoxane (MAO) co-catalyst employed. The presence of $\mathrm{TiO}_{2}$ catalytic traces is below $0.0005 \% \mathrm{w} / \mathrm{w}$, and thus its contribution to dc conductivity is considered unsubstantial. The weight average molecular weight, $M_{\mathrm{w}}$, was determined by rheological measurements to be $M_{\mathrm{w}}=5.6 \cdot 10^{6} \mathrm{~g} / \mathrm{mol}$ [30]. Dodecanethiol functionalized Au nanoparticles $(2-5 \mathrm{~nm}$ in diameter) in toluene $(2 \% \mathrm{w} / \mathrm{v})$ were purchased by Sigma-Aldrich and used as received.

\subsection{Specimen manufacturing}

Dis-UHMWPE powder was suspended in acetone and mixed with the dodecanethiol functionalized gold $(\mathrm{Au})$ nanoparticles in toluene $\left(\mathrm{C}_{7} \mathrm{H}_{8}\right)$ solution 
under magnetic stirring for a few hours, while the solvents were evaporated under a fume hood. The residual solvents were removed by heating the composites at $50^{\circ} \mathrm{C}$ (a temperature below the activation temperature of entanglements formation [11]) under a vacuum for 6 hours. The concentration of gold nanoparticles was set at $1.0 \% \mathrm{w} / \mathrm{w}$. The composite powder was solid-state compacted with a hydraulic press at $125^{\circ} \mathrm{C}$ according to the protocol previously described [11]. The unoriented sample was compacted in a cylindrical disc of $25 \mathrm{~mm}$ diameter and $1.1 \mathrm{~mm}$ thickness, and it is indicated as DR1, while the samples intended for drawing were prepared as rectangular films of $5 \mathrm{~mm} \times 6 \mathrm{~mm}$ with an initial thickness of $1.9 \mathrm{~mm}$ and uniaxially oriented by means of a twin-roll mill at $125^{\circ} \mathrm{C}$ reaching draw ratios of 2 (indicated as sample DR2) and 3 (indicated as sample DR3). The manufacturing process flow is also depicted in Figure 1 below.

\subsection{Characterization}

\section{Raman spectroscopy}

The Raman spectra of the samples were acquired by means of a LabRAM HR provided by Horiba JobinYvon (France) in the wavenumber range of 1500 to $1400 \mathrm{~cm}^{-1}$ at room temperature employing a laser beam at $633 \mathrm{~nm}$.

\section{Vis/NIR spectrophotometer}

The optical absorption of the samples was investigated via a Cary 5000 UV-Vis-NIR spectrophotometer from Agilent Technologies (Santa Clara, California, United States) in the visible wavelength range of 400 to $800 \mathrm{~nm}$ at room temperature.

\section{Dielectric spectroscopy}

The dielectric response of the samples was investigated by means of BDS using an Alpha-N Frequency Response Analyzer. The voltage amplitude of the applied field was kept constant at $3 \mathrm{~V}$, the distance between the electrodes was constant and equal to the thickness of the specimen (the employed dielectric set up ensured the application of a homogeneous electric field), and frequency varied from $10^{0}$ to $10^{4} \mathrm{~Hz}$ employing a two-parallel gold-plated electrode capacitor BDS-1200. The temperature was controlled employing a Novotherm system and was set at $160^{\circ} \mathrm{C}$, with an accuracy of $\pm 0.1^{\circ} \mathrm{C}$, for 21 consecutive frequency sweep cycles with a total duration of approximately 1 hour (1 frequency sweep: $173.5 \mathrm{~s}$ ). Upon reaching the set temperature $\left(160^{\circ} \mathrm{C}\right)$, an equilibrium time of 3 minutes was considered reasonable for reaching thermal equilibrium before the measurement started. Notably, that temperature is measured via a Pt100 thermocouple at the specimen's surface.

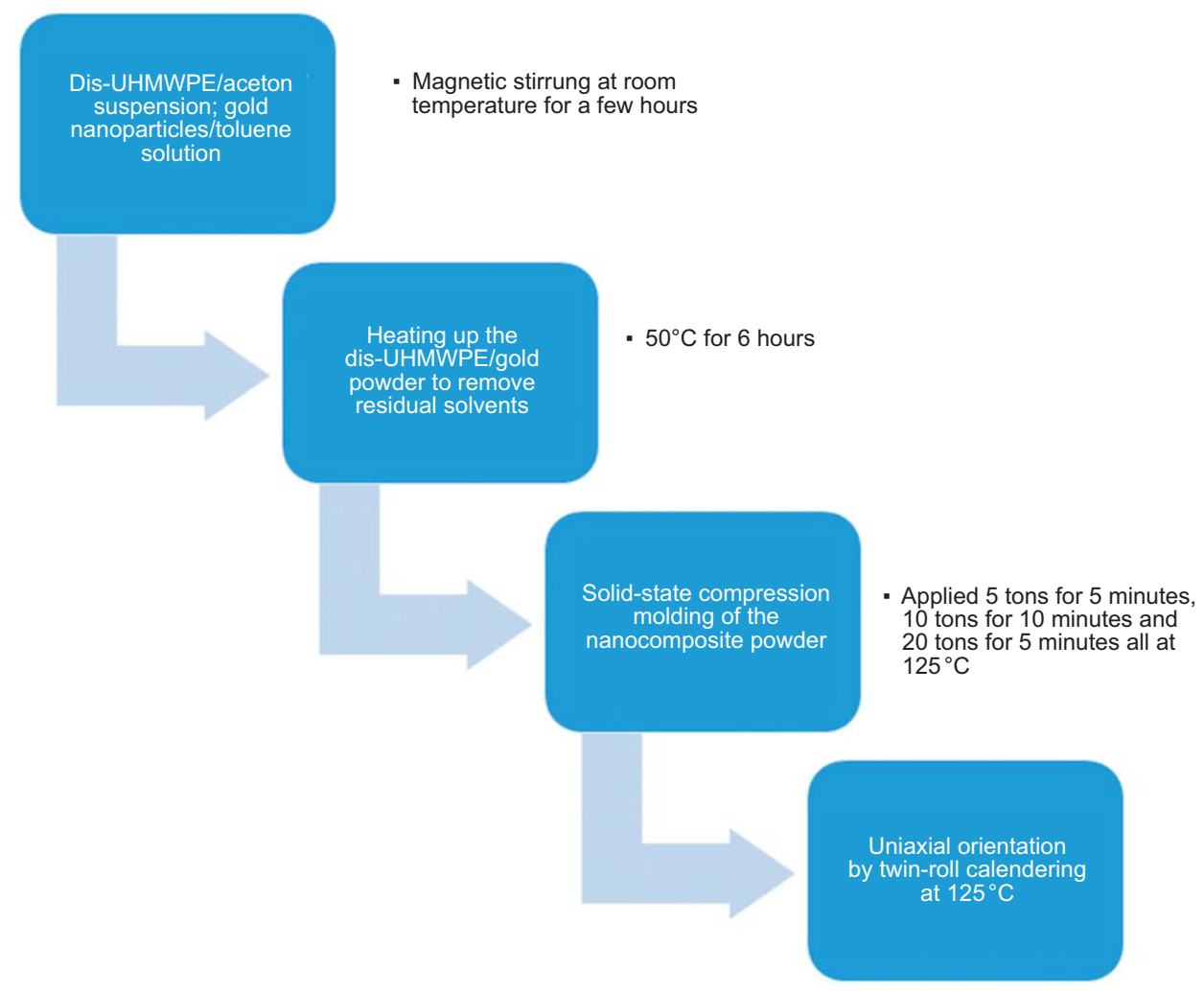

Figure 1. Flow chart of the samples' manufacturing process. 
All the BDS parts were supplied by Novocontrol Technologies (Hundsagen, Germany).

\section{Results and discussion}

The Raman spectra at $30^{\circ} \mathrm{C}$ of the composites are presented in Figure 2a, with the intensities of the peaks changing due to the uniaxial plastic deformation in the examined 1400 to $1500 \mathrm{~cm}^{-1}$ range. The $\mathrm{CH}_{2}$ bending vibrational mode is split into two components at 1440 and $1417 \mathrm{~cm}^{-1}$, indicative of an orthorhombic crystal structure [31]. For the two amorphous peaks, at 1463 and $1440 \mathrm{~cm}^{-1}$ (corresponding to amorphous modes as reported by Strobl and Hagedorn [32]), a decrease in intensity is observed as a function of progressive plastic deformation, while for the $1417 \mathrm{~cm}^{-1}$ crystalline peak, the intensity is found to increase: these observations indirectly indicate an increase in crystallinity due to orientation [33]. In Figure 2b, the optical absorption due to the presence of gold nanoparticles is examined and presented in the visible spectrum at $30^{\circ} \mathrm{C}$. The peak observed at $539 \mathrm{~nm}$ is attributed to the transverse plasmon resonance of the $\mathrm{Au}$ nanoparticles [34], as polyethylene is otherwise transparent in this region. The peak is observed to become narrower with plastic deformation, especially for the case of DR 3 . This is an indication that the average size of gold nanoparticle aggregates is decreasing, with some clusters breaking apart due to orientation. As it can be observed in Figure 2b, the intensities also decrease as a function of draw ratio, as a result of the decreasing thickness with increasing plastic deformation.

The dielectric response of the three samples is presented in Figure 3, where the real part of dielectric permittivity as a function of frequency is shown, increasing the time at $160^{\circ} \mathrm{C}$. As an inset, the imaginary part of dielectric permittivity is also presented. The complex dielectric permittivity $\varepsilon^{*}$ is defined as Equation (1):

$\varepsilon^{*}(\omega)=\varepsilon^{\prime}(\omega)-i \varepsilon^{\prime \prime}(\omega)$

where, $\omega$ is the angular frequency, $\varepsilon^{\prime}$ and $\varepsilon^{\prime \prime}$ are the real and imaginary parts of dielectric permittivity, respectively.

It is observed that the values of the real part of dielectric permittivity increase significantly with frequency decreasing, indicating a strong dipolar polarization for all samples. With the evolution of time, the relaxation tends to lower frequencies, as it can also be observed in the imaginary part of dielectric permittivity, presented as an inset. The loss permittivity versus frequency in the log-log representation shows a linear behavior in the lower frequency range, attributed to the contribution of dc conduction, masking the dipolar polarization peak. An effort is made to remove the conductivity term in order to appreciate the dipolar contribution alone, as seen below.

The frequency-dependent expression of the MaxwellWagner-Sillars (MWS) interfacial polarization phenomenon consists of a Debye dispersion model plus the dc conduction contribution and is given by Equation (2) below in its complex dielectric permittivity representation:

$\varepsilon^{*}(\omega)=\varepsilon_{\infty}+\frac{\varepsilon_{\mathrm{s}}-\varepsilon_{\infty}}{1+i \omega \tau}+\frac{i \sigma_{\mathrm{dc}}}{\varepsilon_{0} \omega^{\mathrm{s}}}$

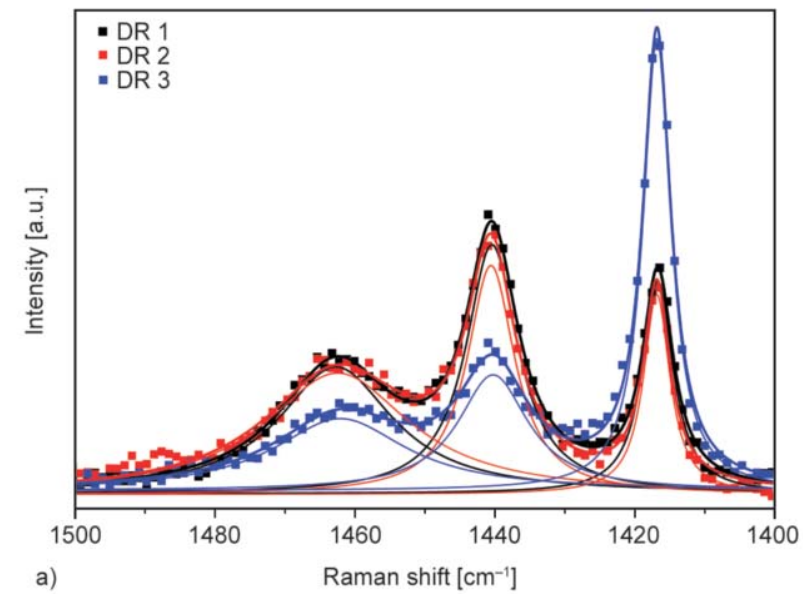

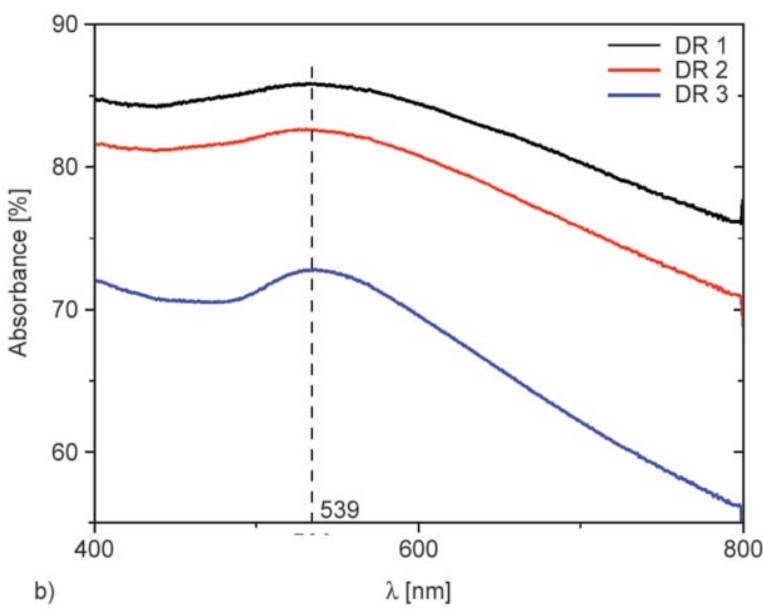

Figure 2. (a) Raman spectra as a function of wavenumber and (b) optical absorbance as a function of wavelength at room temperature varying the draw ratio for the samples under study. 

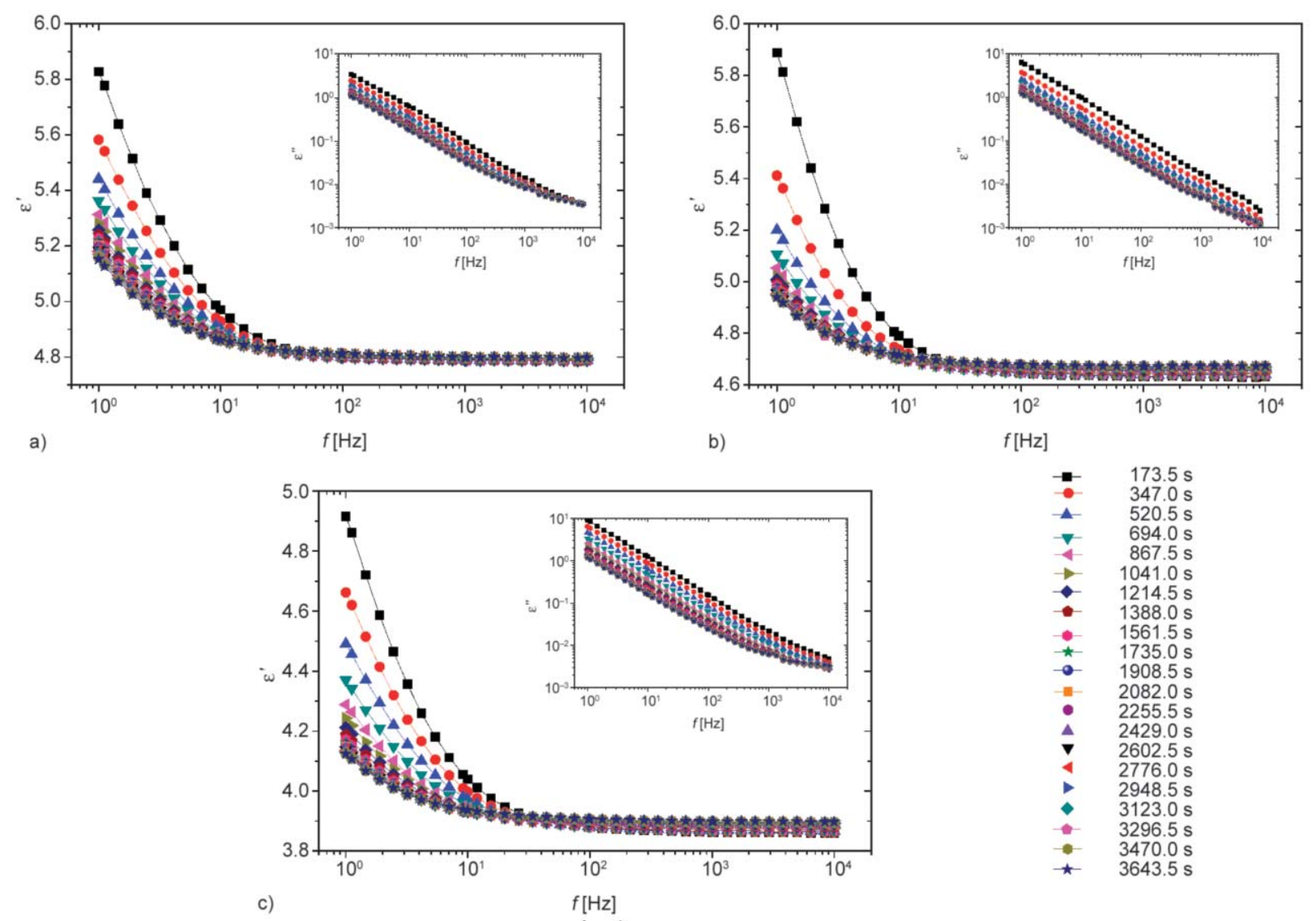

Figure 3. The real part of dielectric permittivity as a function of frequency varying the time for the samples under study: (a) DR 1, (b) DR 2, and (c) DR 3. As an inset, the corresponding imaginary part of dielectric permittivity is presented.

where $\tau$ is the Debye relaxation time, $\lim _{(\mathrm{f} \rightarrow 0)} \varepsilon^{\prime}=\varepsilon_{\mathrm{s}}$, $\lim _{(f \rightarrow \infty)} \varepsilon^{\prime}=\varepsilon_{\infty}, \sigma_{\mathrm{dc}}$ is the $d c$ conduction, $s$ a parameter that depends on the examined material $(0.5 \leq s \leq 1.0)$ and conduction process is considered as Ohmic when $s=1$ while deviations originate because of ionic impurities, electrode polarization, hopping conductivity etc.) [35], and $\varepsilon_{0}$ is the dielectric constant of vacuum $\left(8.854 \cdot 10^{-12} \mathrm{~F} / \mathrm{m}\right)$. In disordered systems, the current density is composed of three additive terms, namely: (i) Ohmic conductivity, (ii) diffusive contribution, and (iii) displacement current density being equal to $\partial D / \partial t$, where $D$ is the electric displacement [36]. At low fields, under steady-state $(\partial D / \partial t=0)$ and constant carrier density in the whole bulk material, Ohmic conductivity appears to be the only contribution. Values of the semi-empirical parameter $s$ tending to unity express the agreement of a system with the aforementioned conditions and the presence of an Ohmic behavior. On the contrary, diffusion current and time-varying fields $(\partial D / \partial t \neq 0)$ contribute to the system's conductivity compensating the Ohmic component and thus leading to lower than unity values of $s$. After implementing Equa- tion (1) in Equation (2), the frequency-dependent expression of the MWS interfacial polarization can be split into a real and an imaginary part, as shown by Equations (3) and (4):

$$
\begin{aligned}
& \varepsilon^{\prime}(\omega)=\varepsilon_{\infty}+\frac{\varepsilon_{\mathrm{s}}-\varepsilon_{\infty}}{1+\omega^{2} \tau^{2}} \\
& \varepsilon^{\prime \prime}(\omega)=\frac{\left(\varepsilon_{s}-\varepsilon_{\infty}\right) \omega \tau}{1+\omega^{2} \tau^{2}}+\frac{\sigma_{\mathrm{dc}}}{\varepsilon_{0} \omega^{\mathrm{s}}}
\end{aligned}
$$

In the case of the imaginary part of dielectric permittivity, the first part of the equation corresponds to the dipolar contribution and the second one to the dc conduction contribution. In materials with relatively high values of electrical conductivity or weak dipolar response, the presence and the migration of charge carriers can mask the dielectric processes and obscure the dielectric analysis. This effect is pronounced especially at low frequencies because the contribution of the conduction term $\left(\sigma_{\mathrm{dc}} \varepsilon_{0}^{-1} \omega^{-s}\right)$ to the loss, permittivity augments with decreasing frequency. Since the dc conduction is present only in the loss permittivity part, following Equations (2)-(4), a way 
to remove the conductivity term, or in other words to determine the dipolar losses, is by applying the logarithmic derivative on the real part of dielectric permittivity as shown in Equation (5) [14]:

$\varepsilon_{\text {der }}^{\prime \prime}=-\frac{\pi}{2} \frac{\partial \varepsilon^{\prime}(\omega)}{\partial \ln \omega}$

thus, making the $\varepsilon_{\text {der }}^{\prime \prime}$ approximately equal only to the dipolar contribution of the imaginary part of dielectric permittivity. Both the $\varepsilon^{\prime}$ and $\varepsilon^{\prime \prime}$ carry equivalent information as they are connected through the Kramers-Kronig relation. Dielectric processes are detected in the spectra of the real and imaginary part of dielectric permittivity in the form of steps and peaks, respectively. Thus, using the real part of permittivity to calculate only the dipolar loss contribution (excluding the contribution of dc conduction) by means of the logarithmic derivative technique is a useful mathematical tool in order to investigate the aforementioned dipolar processes that are shadowed [14]. In Figure 4, the logarithmic derivative of the real part of dielectric permittivity as a function of frequency varying the time is presented. A peak is clearly

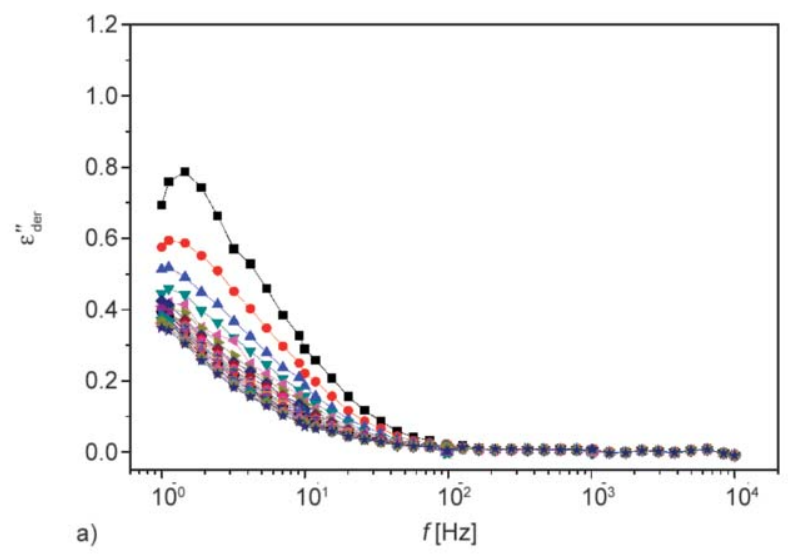

shown for all the samples and is attributed to the dipolar contribution of the MWS interfacial polarization that forms due to the accumulation of charges (mostly electrons) at the interface of the insulator/ conductor system, which in this case is polyethylene/gold nanoparticles [15, 37]. Even at these elevated temperatures, no evidence of the parasitic phenomenon of electrode polarization is observed, as that would give rise to very large capacitances and lead to enormous values of the real and imaginary part of dielectric permittivity.

As can be appreciated from Figure 4, the peak moves to lower frequencies with the evolution of time for all the draw ratios considered. This is in agreement with previous studies of unoriented dis-UHMWPE that showed how, at temperatures above $58^{\circ} \mathrm{C}$, entanglements are energetically favored and is therefore associated with the entanglements formation. The re-entanglement process hinders the achieved polarization by partially reducing the macromolecules' mobility, increasing the relaxation time of the process, and shifting the loss peaks to lower frequencies [11]. In addition, it seems that the relaxation
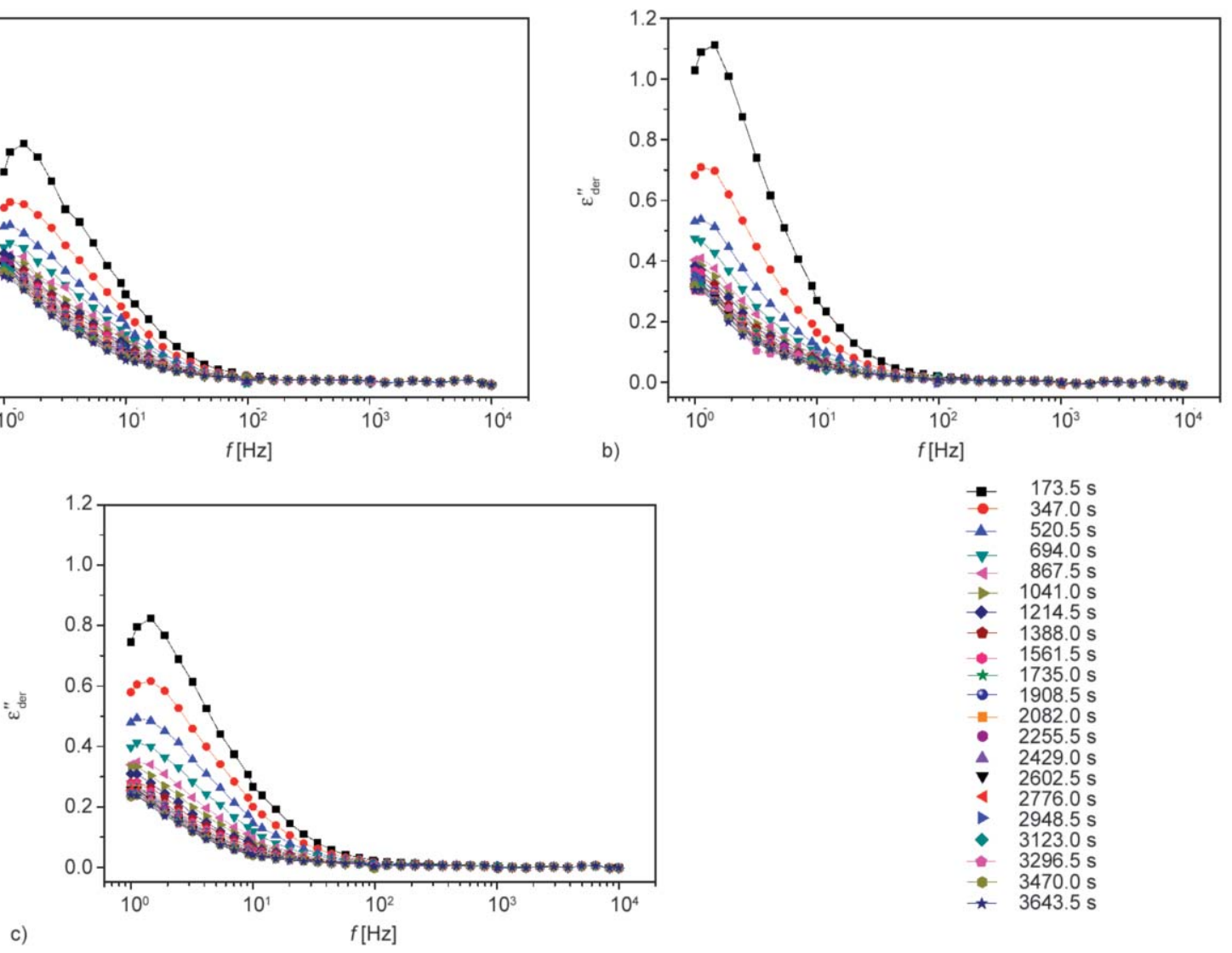

$-\quad 173.5 \mathrm{~s}$

- $\quad 347.0 \mathrm{~s}$

$-\quad 520.5 \mathrm{~s}$

$694.0 \mathrm{~s}$
$-\quad 867.5 \mathrm{~s}$
$-\quad 1041.0 \mathrm{~s}$

$867.5 \mathrm{~s}$
$-\quad 1041.0 \mathrm{~s}$

$1041.0 \mathrm{~s}$

$1388.0 \mathrm{~s}$

$1561.5 \mathrm{~s}$

$1735.0 \mathrm{~s}$ $1908.5 \mathrm{~s}$ $2082.0 \mathrm{~s}$ $2255.5 \mathrm{~s}$ $2429.0 \mathrm{~s}$ $2602.5 \mathrm{~s}$ $2776.0 \mathrm{~s}$ $2948.5 \mathrm{~s}$ $3123.0 \mathrm{~s}$ $3296.5 \mathrm{~s}$ $3470.0 \mathrm{~s}$ $3470.0 \mathrm{~s}$
$3643.5 \mathrm{~s}$

Figure 4. The logarithmic derivative of the real part of dielectric permittivity as a function of frequency (following Equation (5)) varying time for the samples under study: (a) DR 1, (b) DR 2, and (c) DR 3. 
strength (associated with the peak intensity) is decreasing, although since it is shifting towards the lower frequency edge outside the observational window of the experiment, it is not possible to analyze the effect for the overall time frame.

To approximately calculate the dc conductivity, we can subtract the calculated dipolar loss part $\varepsilon_{\text {der }}^{\prime \prime}$ from the total recorded losses $\varepsilon^{\prime \prime}$, as we have shown somewhere else [38] and describe here in Equation (6):

$\varepsilon^{\prime \prime}-\varepsilon_{\mathrm{der}}^{\prime \prime} \approx \frac{\sigma_{\mathrm{dc}}}{\varepsilon_{0} \omega^{\mathrm{s}}} \longleftrightarrow$

$\log \left(\varepsilon^{\prime \prime}-\varepsilon^{\prime \prime}{ }_{\text {der }}\right)=\log \left(\frac{\sigma_{\mathrm{dc}}}{\varepsilon_{0}}\right)-s \log (\omega)$

Equation (6) is a line with a slope equal to $s$ and an intercept equal to $\log \left(\sigma_{\mathrm{dd}} / \varepsilon_{0}\right)$. Equation (6) originates from subtracting the calculated, via Equation (5), values of dipolar losses from the experimentally measured imaginary part of dielectric permittivity. The latter represents the total amount of losses, including all contributions. Since the logarithmic derivative technique removes the contribution of $\mathrm{dc}$ conduction [14], the residual of Equation (6) leads to the calculation de conductivity [38]. Although presented and discussed quantities derive from experimental data providing thus confidence upon their validity, direct experimental evidence is required to show the proximity of calculated and measured values. This is something that is planned to be investigated in a forthcoming study. The corresponding fittings at varying times are given in Figure 5, with $R^{2}$ over 0.992 in any case, indicating the linearity of the obtained data. The fittings were performed only in the frequency range of $10^{0}$ to $10^{1} \mathrm{~Hz}$, where the influence of $d c$ conduction is prominent, in order to exclude any mathematical artifacts at higher frequencies where its contribution vanishes. According to Equation (6), the contribution of $d c$ conduction can be approximately calculated by subtracting the $\varepsilon_{\mathrm{der}}^{\prime \prime}$ from the $\varepsilon^{\prime \prime}$ and by fitting $\log \left(\varepsilon^{\prime \prime}-\varepsilon_{\mathrm{der}}^{\prime \prime}\right)$ versus $\log (\omega)$. The deviation from the Ohmic case $(s=1)$ can be calculated from the slopes of the lines.

In Figure 6, the obtained dc conduction from Equation (6) is presented as a function of time for all the draw ratios under study. From the calculated values of dc conductivity that lie in the order of magnitude of $10^{-10} \mathrm{~S} / \mathrm{m}$, it is evident that the gold nanoparticles are not in contact; hence they do not form a percolating system, as expected from the employed
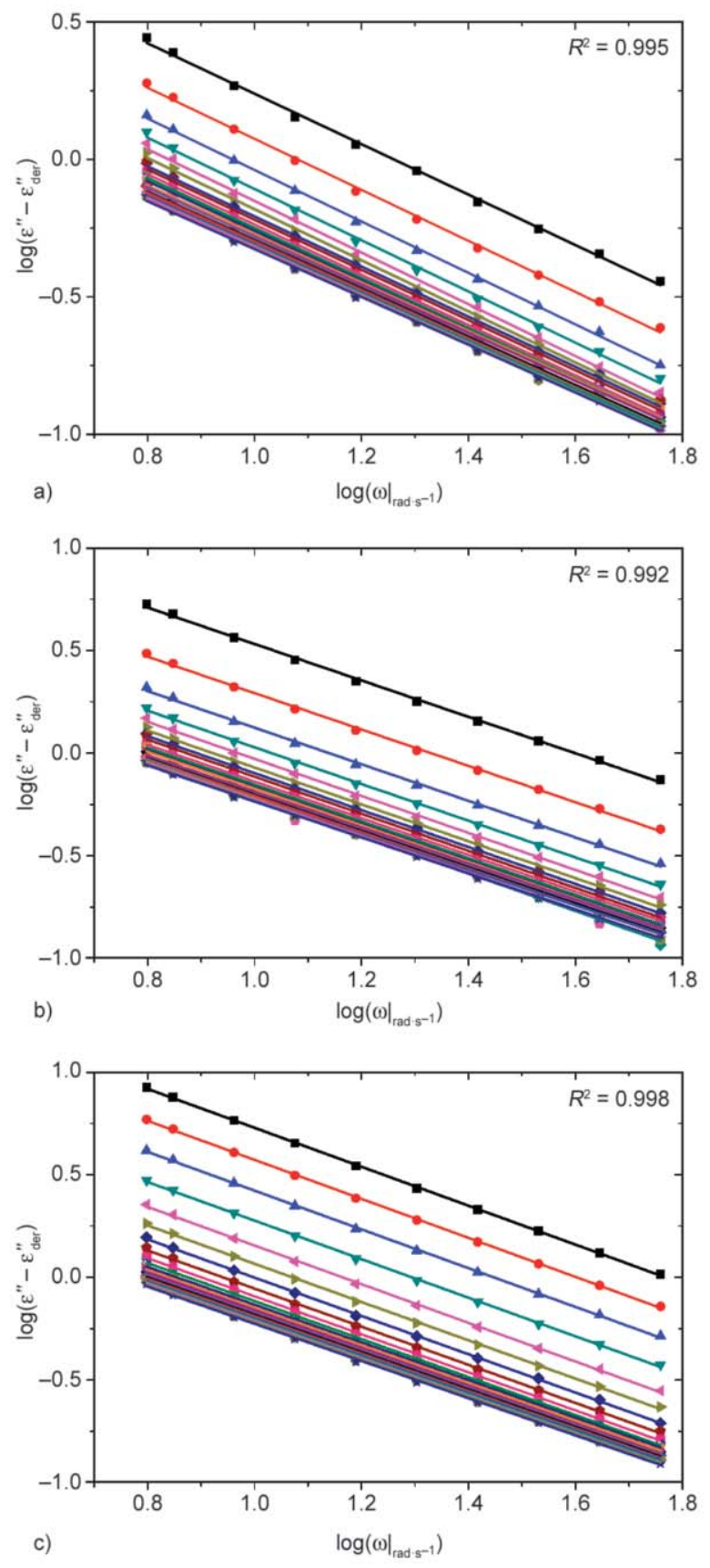

Figure 5. The log-log fittings of the imaginary part minus the logarithmic derivative of dielectric permittivity as a function of frequency (following Equation (6)) for the samples under study: (a) DR 1, (b) DR 2, and (c) DR 3. The minimum $R^{2}$ values are given to highlight the linearity of the obtained data The color of each curve corresponds to the isothermal measurement of time as with Figure 3 and 4.

concentration. It is shown that the electrical conductivity is affected by the formation of entanglements, mainly in the first hour of experiments; after this time, a plateau region is achieved. Considering that the measurement takes place above the melting temperature, the initial dc conductivity difference between the samples is attributed to partially oriented 


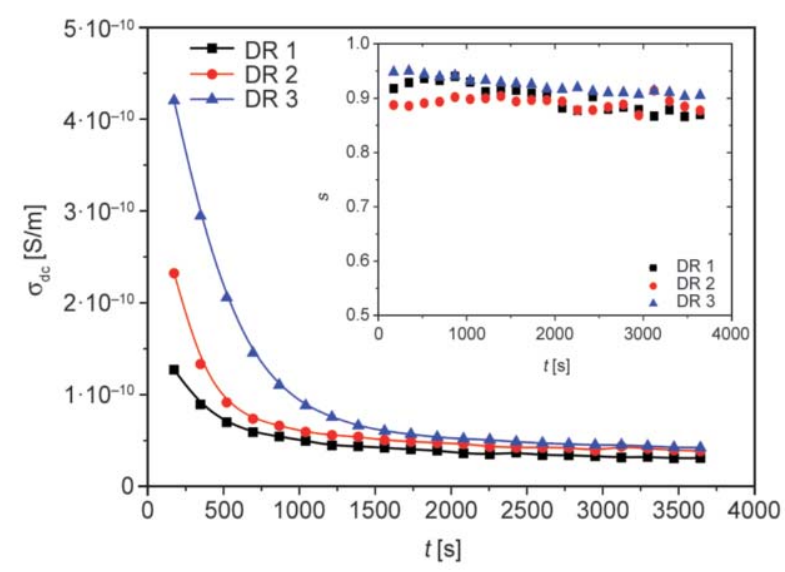

Figure 6. The calculated values of the dc conductivity (the lines serve as guides for the eye only) with the parameter s being included as an inset from Equation (6).

amorphous segments that, over time lose their orientation and thus approach a plateau value. The deviation of parameter $s$ from 1 can be considered as a deviation from the Ohmic behavior and is attributed to the hopping conductivity of the charger carriers due to the presence of the gold nanoparticles [39, 40]. In the inset of Figure 6, it can be observed that for all the studied samples, there is a slight decrease of the parameter $s$ over time, indicating further deviation from the Ohmic behavior that is related to the increase of disorder with the formation of entanglements and the loss of orientation.

According to previous dielectric studies on disUHMWPE that we have conducted [11], the real part of electric modulus as a function of time shows an increase (build-up) similar to what was observed for the real part of mechanical modulus from melt plateplate rheology due to the progressive formation of entanglements. The complex electric modulus follows the relation below (Equation (7)):

$$
\begin{aligned}
M^{*}(\omega) & =\frac{1}{\varepsilon^{*}(\omega)}= \\
& =\frac{\varepsilon^{\prime}(\omega)}{\varepsilon^{\prime}(\omega)^{2}+\varepsilon^{\prime \prime}(\omega)^{2}}+i \frac{\varepsilon^{\prime \prime}(\omega)}{\varepsilon^{\prime}(\omega)^{2}+\varepsilon^{\prime \prime}(\omega)^{2}}= \\
& =M^{\prime}(\omega)+i M^{\prime \prime}(\omega)
\end{aligned}
$$

where $M^{\prime}$ and $M^{\prime \prime}$ correspond to the real and imaginary parts of electric modulus, respectively. Considering the equation that was first proposed by Yamazaki et al., [41] and Teng et al., [42] to follow chain entanglement and relaxation in the rheological build up data or crystallization experiments and employed by

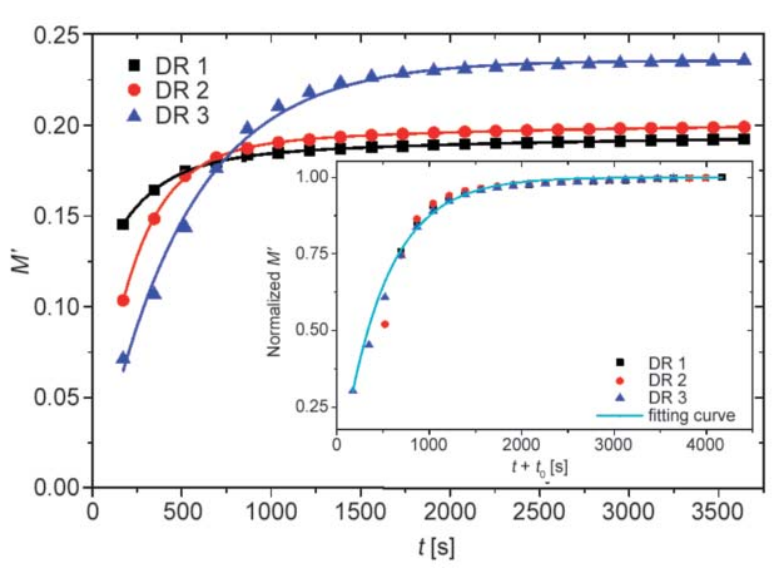

Figure 7. The real part of electric modulus (the lines correspond to fittings following Equation (8)) both as a function of time for the samples under study at $\omega=$ $9.15 \mathrm{rad} / \mathrm{s}$. As an inset, a normalized representation of the same data is provided with a common fitting as a superposition of extended time $\left(t+t_{0}\right)$ and draw ratio with DR 3 being the reference sample $\left(t_{0}=0 \mathrm{~s}\right)$. The $t_{0}$ parameter for samples DR 1 and DR 2 is 520.5 and $347.0 \mathrm{~s}$, respectively. All the parameters are presented in Table 1.

Liu et al., [12] for dis-UHMWPE, we have employed it to fit the electric modulus build-up. The fitted values are presented in Figure 7, and the expression can be seen below (Equation (8)):

$M^{\prime}(t)=M_{0}^{\prime}-\sum_{\mathrm{i}=1}^{\mathrm{N}} M_{\mathrm{i}}^{\prime} \mathrm{e}^{\left(-\frac{t}{\tau_{\mathrm{i}}}\right)}$

where $M^{\prime}(t)$ is the variation of the real part of electric modulus with time, $M^{\prime}{ }_{0}$ is the plateau value of the real part of electric modulus, $M_{\mathrm{i}}^{\prime}$ is an electric modulus increment corresponding to a characteristic time $\tau_{\mathrm{i}}$. In the inset of Figure 7, the normalized $M^{\prime}$ data as a function of time for the investigated samples are presented by dividing the $M^{\prime}(t)$ with the plateau value $\left(M_{0}^{\prime}\right)$, and their corresponding times shifted by $t_{0}$ to create a master curve.

The reported findings in Figure 7 agree with the literature [12] on the two time-regions of the formation of entanglements at a very similar angular frequency. The first drastic change is considered to be the chain explosion, i.e., the formation of entanglements in the disentangled amorphous phase that in the solid state consisted of the pristine crystalline domains. The slower change is due to chain reptation and further entanglements, as shown with both rheological experiments and DSC. Those experiments showed that the low-temperature endothermic peak, associated with crystalline structures formed in the entangled amorphous phase, increases with the annealing time 
Table 1. Presented are the fitting parameters for the real part of electric modulus as a function of time, according to Equation (8) employing two modes. The fitted superimposed data in the inset of Figure 7 are also included.

\begin{tabular}{|l|c|c|c|c|c|c|}
\hline \multicolumn{1}{|c|}{ Sample } & $\boldsymbol{M}_{\mathbf{0}}$ & $\boldsymbol{M}^{\prime}{ }_{\mathbf{1}}$ & $\begin{array}{c}\boldsymbol{\tau}_{\mathbf{1}} \\
{[\mathbf{s}]}\end{array}$ & $\boldsymbol{M}_{\mathbf{2}}{ }^{\prime}$ & $\begin{array}{c}\boldsymbol{\tau}_{\mathbf{2}} \\
{[\mathbf{s}]}\end{array}$ & $\boldsymbol{R}^{\mathbf{2}}$ \\
\hline DR 1 & 0.194 & 0.071 & 250.0 & 0.015 & 1783.1 & 0.99992 \\
\hline DR 2 & 0.201 & 0.179 & 227.8 & 0.015 & 1680.6 & 0.99979 \\
\hline DR 3 & 0.236 & 0.242 & 496.9 & - & - & 0.99391 \\
\hline Superimposed & 1.000 & 1.000 & 483.1 & - & - & 0.97149 \\
\hline
\end{tabular}

[12]. However, the timescales involved in the rheological build-up experiments are significantly higher than the dielectric experiments presented here (i.e. in the range of an order of magnitude).

Interestingly, if the values of electric modulus presented in Figure 7 were subject to normalization, then a similar concept as the time-temperature-superposition principle could be applied, but instead of temperature, draw ratio can be employed. The specific analysis presented in the inset of Figure 7 gives further insights on the chain dynamic in dis-UHMWPE: it appears that orientation hinders the formation of entanglements over time at the initial stages of melt state (before equilibrium), in a way that resembles what happens to unoriented dis-UHMWPE samples of higher molecular weights, as discussed in the literature [43]. This could be an interesting tool to predict the properties of oriented samples at different draw ratios, and maybe it could be combined with similar testing at different temperatures.

Comparing the characteristic times, $\tau$, as obtained from fittings of the rate at which the low-temperature endothermic peak increases with the annealing time of a similar dis-UHMWPE sample in the literature [12] and our data in Table 1, it is visible that the chain explosion corresponds to our $2^{\text {nd }}$ mode $\left(\tau_{2}\right)$. Considering that the real part of electric modulus 'carries' both information from the dipolar response and the dc conduction (a combination of Equations (2) and (7)) variation in either affects its values. The effect of time in the formation of entanglements has been indeed observed in both the dipolar (Figure 4) and the $d c$ (Figure 6) response as discussed earlier. The $1^{\text {st }}$ mode corresponds to the first 3 to 5 minutes of experiments. It might be associated with entanglements forming in the unentangled areas that previously were part of the extended nascent crystals and/or partially oriented amorphous parts of the polymer chain. This notion also agrees with the evidence presented in Figure 6, where a stronger decrease in $d c$ conductivity is observed as the draw ratio increases since the crystallinity increases with orientation, as presented in Figure 2a.

\section{Conclusions}

Broadband dielectric spectroscopy (BDS) has been employed to understand the formation of entanglements as a function of draw ratio during the melting of oriented dis-UHMWPE/gold nanocomposites. The dc conduction values are approximately calculated by subtracting the logarithmic derivative of dielectric permittivity from the loss permittivity values, and they are found to decrease with the formation of entanglements. This effect is more intense when the draw ratio increases due to the initial entanglements forming in the molten unentangled crystalline regions and the partially oriented amorphous segments in the oriented samples. The dc conductivity of all the samples was observed to be non-purely Ohmic, attributed to the hopping mechanism due to the presence of gold nanoparticles, and was observed to deviate further from the Ohmic behavior with time, as a result of the entanglements formation and loss of orientation. In addition, with the help of the logarithmic derivative of dielectric permittivity, a strong peak is obtained in the absence of dc conductivity, attributed to the dipolar contribution of the MWS interfacial polarization between the gold nanoparticles and the polymer chains. With the progression of time, the relaxation time of the MWS interfacial polarization increases, indicating the formation of entanglements. Finally, with increasing draw ratio, it was observed that the formation of entanglements is hindered, drawing a parallel with unoriented samples of increasing molecular weight. This concept could potentially be used as a tool to predict the properties of oriented samples of dis-UHMWPE.

\section{Acknowledgements}

This project has been funded by the Engineering and Physical Science Research Council (EPSRC), grant EP/ K034405/1.

The authors would like to thank Dr. Giuseppe Forte for the synthesis of the dis-UHMWPE powder and Dr. Gianfranco Claudio for providing access to the Vis/NIR spectrophotometry based at Centre for Renewable Energy Systems (CREST), Loughborough University. 


\section{References}

[1] Ronca S., Forte G., Tjaden H., Yao Y., Rastogi S.: Tailoring molecular structure via nanoparticles for solventfree processing of ultra-high molecular weight polyethylene composites. Polymer, 53, 2897-2907 (2012). https://doi.org/10.1016/j.polymer.2012.04.051

[2] Rendell R. W., Ngai K. L., McKenna G. B.: Molecular weight and concentration dependences of the terminal relaxation time and viscosity of entangled polymer solutions. Macromolecules, 20, 2250-2256 (1987). https://doi.org/10.1021/ma00175a033

[3] Rastogi S., Lippits D. R., Peters G. W. M., Graf R., Yao Y., Spiess H. W.: Heterogeneity in polymer melts from melting of polymer crystals. Nature Materials, 4, 635641 (2005).

https://doi.org/10.1038/nmat1437

[4] Drakopoulos S. X., Forte G., Ronca S.: Relaxation dynamics in disentangled ultrahigh molecular weight polyethylene via torsional rheology. Industrial and Engineering Chemistry Research, 59, 4515-4523 (2020). https://doi.org/10.1021/acs.iecr.9b06401

[5] Pandey A., Toda A., Rastogi S.: Influence of amorphous component on melting of semicrystalline polymers. Macromolecules, 44, 8042-8055 (2011). https://doi.org/10.1021/ma201797k

[6] Wunderlich B., Czornyj G.: A study of equilibrium melting of polyethylene. Macromolecules, 10, 906-913 (1977). https://doi.org/10.1021/ma60059a006

[7] Rastogi S., Lippits D. R., Höhne G. W. H., Mezari B., Magusin P. C. M. M.: The role of the amorphous phase in melting of linear UHMW-PE; Implications for chain dynamics. Journal of Physics: Condensed Matter, 19, 205122/1-205122/21 (2007).

https://doi.org/10.1088/0953-8984/19/20/205122

[8] Rastogi S., Yao Y., Lippits D. R., Höhne G. W. H. H., Graf R., Spiess H. W., Lemstra P. J.: Segmental mobility in the non-crystalline regions of semicrystalline polymers and its implications on melting. Macromolecular Rapid Communications, 30, 826-839 (2009). https://doi.org/10.1002/marc.200900025

[9] Romano D., Tops N., Andablo-Reyes E., Ronca S., Rastogi S.: Influence of polymerization conditions on melting kinetics of low entangled UHMWPE and its implications on mechanical properties. Macromolecules, 47, 4750-4760 (2014).

https://doi.org/10.1021/ma5008122

[10] Ronca S., Romano D., Forte G., Andablo-Reyes E., Rastogi S.: Improving the performance of a catalytic system for the synthesis of ultra high molecular weight polyethylene with a reduced number of entanglements. Advances in Polymer Technology, 31, 193-204 (2012). https://doi.org/10.1002/adv.21265

[11] Drakopoulos S. X., Psarras G. C., Forte G., MartinFabiani I., Ronca S.: Entanglement dynamics in ultrahigh molecular weight polyethylene as revealed by dielectric spectroscopy. Polymer, 150, 35-43 (2018). https://doi.org/10.1016/j.polymer.2018.07.021
[12] Liu K., de Boer E. L., Yao Y., Romano D., Ronca S., Rastogi S.: Heterogeneous distribution of entanglements in a nonequilibrium polymer melt of UHMWPE: Influence on crystallization without and with graphene oxide. Macromolecules, 49, 7497-7509 (2016). https://doi.org/10.1021/acs.macromol.6b01173

[13] Schönhals A., Kremer F.: Analysis of dielectric spectra. in 'Broadband dielectric spectroscopy' (eds.: Kremer F., Schönhals A.) Springer, Berlin, 59-98 (2003). https://doi.org/10.1007/978-3-642-56120-7

[14] Wübbenhorst M., van Turnhout J.: Analysis of complex dielectric spectra. I. One-dimensional derivative techniques and three-dimensional modelling. Journal of Non-Crystalline Solids, 305, 40-49 (2002). https://doi.org/10.1016/S0022-3093(02)01086-4

[15] Tsangaris G. M., Psarras G. C., Kouloumbi N.: Electric modulus and interfacial polarization in composite polymeric systems. Journal of Materials Science, 33, 20272037 (1998).

https://doi.org/10.1023/A:1004398514901

[16] Gerhardt R.: Impedance and dielectric spectroscopy revisited: Distinguishing localized relaxation from longrange conductivity. Journal of Physics and Chemistry of Solids, 55, 1491-1506 (1994). https://doi.org/10.1016/0022-3697(94)90575-4

[17] Rytöluoto I., Gitsas A., Pasanen S., Lahti K.: Effect of film structure and morphology on the dielectric breakdown characteristics of cast and biaxially oriented polypropylene films. European Polymer Journal, 95, 606-624 (2017).

https://doi.org/10.1016/j.eurpolymj.2017.08.051

[18] Green C. D., Vaughan A. S., Mitchell G. R., Liu T.: Structure property relationships in polyethylene/montmorillonite nanodielectrics. IEEE Transactions on Dielectrics and Electrical Insulation, 15, 134-143 (2008). https://doi.org/10.1109/T-DEI.2008.4446744

[19] Lau K. Y., Vaughan A. S., Chen G.: Nanodielectrics: Opportunities and challenges. IEEE Electrical Insulation Magazine, 31, 45-54 (2015). https://doi.org/10.1109/MEI.2015.7126073

[20] Zhong S-L., Dang Z-M., Zhou W-Y., Cai H-W.: Past and future on nanodielectrics. IET Nanodielectrics, 1 , 41-47 (2018). https://doi.org/10.1049/iet-nde.2018.0004

[21] Kang D., Wang G., Huang Y., Jiang P., Huang X.: Decorating $\mathrm{TiO}_{2}$ nanowires with $\mathrm{BaTiO}_{3}$ nanoparticles: A new approach leading to substantially enhanced energy storage capability of high-k polymer nanocomposites. ACS Applied Materials and Interfaces, 10, 4077-4085 (2018). https://doi.org/10.1021/acsami.7b16409

[22] Reddy A. L. M., Gowda S. R., Shaijumon M. M., Ajayan P. M.: Hybrid nanostructures for energy storage applications. Advanced Materials, 24, 5045-5064 (2012). https://doi.org/10.1002/adma.201104502 
[23] Park B., Im K-J., Cho K., Kim S.: Electrical characteristics of gold nanoparticle-embedded MIS capacitors with parylene gate dielectric. Organic Electronics: Physics, Materials, Applications, 9, 878-882 (2008). https://doi.org/10.1016/j.orgel.2008.06.010

[24] Choy C. L.: Thermal conductivity of polymers. Polymer, 18, 984-1004 (1977).

https://doi.org/10.1016/0032-3861(77)90002-7

[25] Choy C. L., Greig D.: The low-temperature thermal conductivity of a semi-crystalline polymer, polyethylene terephthalate. Journal of Physics C: Solid State Physics, 8, 3121-3130 (1975).

https://doi.org/10.1088/0022-3719/8/19/012

[26] Ronca S., Igarashi T., Forte G., Rastogi S.: Metalliclike thermal conductivity in a lightweight insulator: Solid-state processed ultra high molecular weight polyethylene tapes and films. Polymer, 123, 203-210 (2017). https://doi.org/10.1016/j.polymer.2017.07.027

[27] Robbins A. B., Drakopoulos S. X., Martin-Fabiani I., Ronca S., Minnich A. J.: Ballistic thermal phonons traversing nanocrystalline domains in oriented polyethylene. Proceedings of the National Academy of Sciences, 116, 17163-17168 (2019). https://doi.org/10.1073/pnas.1905492116

[28] Shen S., Henry A., Tong J., Zheng R., Chen G.: Polyethylene nanofibres with very high thermal conductivities. Nature Nanotechnology, 5, 251-255 (2010). https://doi.org/10.1038/nnano.2010.27

[29] Forte G., Ronca S.: Synthesis of disentangled ultra-high molecular weight polyethylene: Influence of reaction medium on material properties. International Journal of Polymer Science, 2017, 7431419/1-7431419/8 (2017). https://doi.org/10.1155/2017/7431419

[30] Ronca S., Forte G., Tjaden H., Rastogi S.: Solvent-free solid-state-processed tapes of ultrahigh-molecularweight polyethylene: Influence of molar mass and molar mass distribution on the tensile properties. Industrial and Engineering Chemistry Research, 54, 7373-7381 (2015).

https://doi.org/10.1021/acs.iecr.5b01469

[31] Boerio F. J., Koenig J. L.: Raman scattering in crystalline polyethylene. The Journal of Chemical Physics, 52, 3425-3431 (1970). https://doi.org/10.1063/1.1673506

[32] Strobl G. R., Hagedorn W.: Raman spectroscopic method for determining the crystallinity of polyethylene. Journal of Polymer Science: Polymer Physics Edition, 16, 1181-1193 (1978).

https://doi.org/10.1002/pol.1978.180160704

[33] Drakopoulos S. X., Tarallo O., Guan L., Martin-Fabiani I., Ronca S.: Nanocomposites of Au/disentangled UHMWPE: A combined optical and structural study. Molecules, 25, 3225/1-3225/14 (2020).

https://doi.org/10.3390/molecules25143225
[34] Norman T. J., Grant C. D., Magana D., Zhang J. Z., Liu J., Cao D., Bridges F., van Buuren A.: Near infrared optical absorption of gold nanoparticle aggregates. The Journal of Physical Chemistry B, 106, 7005-7012 (2002).

https://doi.org/10.1021/jp0204197

[35] Hartmann L., Fukao K., Kremer F.: Molecular dynamics in thin polymer films. in 'Broadband dielectric spectroscopy’ (eds.: Kremer F., Schönhals A.) Springer, Berlin, 433-473 (2003).

https://doi.org/10.1007/978-3-642-56120-7

[36] Böttger H., Bryskin V. V.: Hopping conduction in solids. Akademie-Verlag, Berlin (1985).

[37] Ezquerra T. A., Canalda J. C., Sanz A., Linares A.: On the electrical conductivity of PVDF composites with different carbon-based nanoadditives. Colloid and Polymer Science, 292, 1989-1998 (2014).

https://doi.org/10.1007/s00396-014-3252-6

[38] Drakopoulos S. X., Karger-Kocsis J., Psarras G. C.: The effect of micro-fibrillated cellulose upon the dielectric relaxations and DC conductivity in thermoplastic starch bio-composites. Journal of Applied Polymer Science, 137, 49573/1-49573/10 (2020). https://doi.org/10.1002/app.49573

[39] Psarras G. C.: Hopping conductivity in polymer matrix-metal particles composites. Composites Part A: Applied Science and Manufacturing, 37, 1545-1553 (2006).

https://doi.org/10.1016/j.compositesa.2005.11.004

[40] Connor M. T., Roy S., Ezquerra T. A., Baltá Calleja F. J.: Broadband AC conductivity of conductor-polymer composites. Physical Review B, 57, 2286-2294 (1998). https://doi.org/10.1103/PhysRevB.57.2286

[41] Yamazaki S., Gu F., Watanabe K., Okada K., Toda A., Hikosaka M.: Two-step formation of entanglement from disentangled polymer melt detected by using nucleation rate. Polymer, 47, 6422-6428 (2006). https://doi.org/10.1016/j.polymer.2006.07.010

[42] Teng C., Gao Y., Wang X., Jiang W., Zhang C., Wang R., Zhou D., Xue G.: Reentanglement kinetics of freezedried polymers above the glass transition temperature. Macromolecules, 45, 6648-6651 (2012).

https://doi.org/10.1021/ma300885w

[43] Talebi S., Duchateau R., Rastogi S., Kaschta J., Peters G. W. M., Lemstra P. J.: Molar mass and molecular weight distribution determination of UHMWPE synthesized using a living homogeneous catalyst. Macromolecules, 43, 2780-2788 (2010).

https://doi.org/10.1021/ma902297b 